\title{
Reanalysis of Foxconn Incident Based On the Perspective of Group Dynamics Theory
}

\author{
Feng Zhao, Yanying Tang, Yawen Gan \\ Research Center for Human Resource and Education Development, School of Public Administration, \\ University of International Business and Economics, Beijing 100029, China
}

Key words: Group dynamics theory, "Economic man" Hypothesis, Media guidance, Personal factors.

\begin{abstract}
Since the beginning of 2010, a series of employees committed suicide in Foxconn Group which has aroused widespread concern in the society. Many scholars have studied the incident from different perspectives. Based on the theory of group dynamics of German psychologist Levin, this paper explores the causes of the Foxconn event from the perspective of individual and social environment of employees, and tries to evoke the relationship between the enterprise and the related subject and the surroundings the role of attention and attention.
\end{abstract}

\section{Introduction}

Since the beginning of 2010,in Foxconn Group, a series of employees had committed suicide which had caused widespread concern in the society, Foxconn Group actively dealed with such incidents, succeeded to settle such management issues timely and effectively by taking active measures and improvement of the management measures. Through the analysis of the incident,I found that this was not only the problem of the Foxconn management at the time but also involved the media, individual employee and other factors.I hope that this research can bring practical warning and reference for the field of management practice.

\section{Group dynamics theory}

The group dynamics theory was first proposed by the German psychologist Kurt Lewin, who argued that human psychology and behavior depending on the interaction between the internal needs and the surroundings. When the human needs can not been meted, There will be a internal force field tension, and the surrounding environment factors play a role in the fuse. Human behavior depends on the interaction between the internal force field and the situational force field (environmental factors), and the main determinant is the tension of the internal force field. According to the theory of "field", Levin proposed the behavior formula: $B=f(P * E) .{ }^{[1]}$ where $B$ is the behavior, $P$ is the individual, $E$ is the environment, and the behavior is a function of the individual and the environment.

Since the employees of Foxconn jumping incident happened,many scholars conducted in-depth analysis of the behavior from different perspectives.For example, the doctoral student of the School of Politics and Public Administration of Wuhan University, Meilin, explored the formation of corporate management culture from the perspective of humanities and called on the society to give more attention to the employees of contemporary enterprises based on this incident. For example, Yi Lingfeng and Guoying Ting East China Normal University, on account of the Foxconn improving the salary of $66 \%$ of the "comfort" measures after the event of 12 consecutive jumping, discussed that the modern enterprise should find the balance point between the economic contract and psychological contract; another example, Hunan First Normal College Luo Weiguang and Zhu Jing ,Taking the 
suicide incident of Foxconn employees as the breakthrough point, this paper analyzed that the media should pay attention to the psychological demands of the relevant groups and adhere to the healthy communication in the psychological crisis reports as the core position.By combing the literature, I found that no scholars have studied the frequent suicide of Foxconn employees from the perspective of Levine Group dynamics theory. Therefore, based on the integration and utilization of existing data, this paper analyzes the suicidal behavior of Foxconn employees from the two dimensions of situational force field (environment) and internal force field (individual).

\section{Environmental Factor Analysis (E)}

\section{An Analysis of the Working Environment Based on the Hypothesis of "Economic Man"}

Gou Tainming and Foxconn, a book indicated that the 24 hours shift of Foxconn's production model,mass production,efficient operation of the process, emphasising on customers winning initiative advantages as the main features. The way of doing business was evaluated as a tiger-like vicious, fox-like cunning. Thus, Foxconn's corporate culture is called "Tiger Fox" cultural management model [2].

"Tiger-like vicious" is mainly reflected in the strict discipline of Foxconn, strict management, pay (paying) attention to obedience, tough style of semi-militarized management. First of all, in the management concept level, Foxconn's production line staff need to follow not only the strict militarization of training and rules in the entry and meeting, but also accept a variety of implicit rules. For example, everywhere you can see uncomfortable warning, the president instructions, cultural slogans. Secondly, in the management model level, Foxconn uses the "scientific management" model, the work has been specificized with regular process, and the staff becomes a machine, lack of the humanistic concerns as an independent individual. Again, in the management of organizational structure level, Foxconn factory have a hierarchical pyramid structure. Line workers for the bottom, and then the line leader, step by step layers up. Managers are divided into the relevant level that is directly linked to the salary with slowly promtion Finally, at the management system level, strictly "review". Foxconn recruited a special group of employees every year, called the xingan class, members from the domestic elite graduates, the group was focused on training. In accordance with the provisions the group members must first go to the production line for 3-6 months internship,during which they must accept the supervisor "review".

"Fox-like cunning" is mainly reflected in Foxconn's original pay system. First of all, Foxconn factory is labor-intensive enterprises, its significant advantage is cheap labor. For example, before the jumping incident, the Shenzhen Foxconn factory have 400,000 employees, of which the annual salary are about ten thousand accounted for $90 \%$, annual salary of more than 120,000 yuan only $0.8 \%$. [3] Secondly, need to work overtime. Foxconn workers have to work 60 hours a week to earn about $\$ 500$. As a result, Foxconn employees had to choose to sign overtime agreements and fulfill the terms of the agreement in exchange for overtime paying beyond normal working hours (12 hours / day). Thus, the salary of Foxconn employees generally consists of the minimum basic salary and the occasional overtime paying per month. Finally, overtime working normalization, employees even eager to get overtime working chances through the hidden rules and complain about lacking of overtime working off-season, and gradually ignored the demand of themselves as "social people" .In addition, Foxconn staff flow quickly, employees are facing the pressure of cut-throat competition.

Foxconn's main management model can be classified as "Taylor system", that "economic man" hypothesis is for the management of theoretical basis: from the hedonistic philosophy point of view that all human behavior is to maximize their own interests. [4] on account of this assumption of management principles and measures it is the mechanical management model based on money and power-based, specifically it can be attributed to the following three aspects: First, the management focus is to improve production efficiency and complete production tasks, ignoring workers emotion. Second, the management work is only for a few people, workers only to obey the management rules; 
Finally, implement clear reward and punishment measures; the reward policy mainly rely on money to motivate employees;severe punishment to the deliberately going slow ; it is the typical "carrot plus sticks" policy.

Therefore, from the perspective of benifit, some of the management experience is desirable for helping Foxconn successfully enter the world's top 500 foundry. but from the perspective of labor relations, they see the staff as a tool to achieve profit, re-system, rewards Penalty, lack of respect and care, this management system and organizationalculture and other consist of the soft environment which is closely related to the Foxconn employee junping incident. in other words it is one of the fuse factors as the group dynamics theory said.

\section{Lack of positive guidance for media environment analysis}

\section{Continuous negative media report indirectly induced "infectious" suicide}

In early 2010, from the first case of Foxconn employees suicide to the final jumping, a series of media report articles lead to the public questioning and thinking of what a role the media played in the tragedy. Among them, there are views saying that "it is the media reports that 'induced' adverse effects. This led to a fierse discussion on whether it is right or not to adhere to the 'journalism professionalism' between academia and the industry nationwide. [5] Tracking hot topics is a professional instinct for a media man. But for the audience, this is not only just a news, but also the driving force of its behavior. This kind of social public opinion environment will become an important fuse to"infectious suicide", and then a terrible "Witt effect."

Foxconn employees are mostly borned in 1980s and 1990s. when they lose the protection of their parents,start to work independently especially in the Foxconn factory, facing such high-intensity work, there will be a significant psychological gap, and precisely they are not fully prepared for this psychological transition. In the face of serious disproportionate pay and in come, mechanization work, unjustified accusations from the higher-up their negative emotions continue to grow and continue to spread effectively without any ways to release. first of all, no one cares about the psychological states of Foxconn employees ) ; Secondly, there is an instinctive exclusion for people to see the psychological physician for a long time.

Searching for the media reports about Foxconn jumping incident mostly of which were negative criticisms and even saying that Foxconn was a "sweatshop". these reports may increase the pity feelings of the public ; but for the employees to continue to work for the Foxconn, only Deepen dissatisfaction, A combination of the negative reports about the jumping incident and lack of psychological guidance of the staff is definitely to blame for this continuous tragedy.

Media's over-concern promote the public for audience psychological

The media "demonization" reports about Foxconn suicide incident, making the ordinary people after see Foxconn "six jump" "seven jump" "eight jump", the first idea coming to mind is "when the ninth will appear ". Obviously, this has deviated from the normal concern about news events.

A series of reports about the Foxconn incident lacked objectivity, and in the face of tragedy, the public became insensitive spectators. The media in order to attract the public attentions and even choose to stay outside Foxconn to report a tragedy, into a vicious circle. According to the formula of $\mathrm{B}=\mathrm{f}(\mathrm{P} * \mathrm{E})$, the employee suicide incident and employee's working environment (mainly refers to the soft environment of the enterprise, not the hardware working environment) and the public opinion Environment formed by the media after the incident are closely related.

\section{Personal Factor Analysis (P)}

According to Levin's theory of group dynamics, the suicide incident of Foxconn employees not only related to environmental factors, but also inseparable from personal factors. Shenzhen Longhua Foxconn Chief Executive Officer Li Jinming once described the two generations of Chinese mainland workers. He believes that the first generation of migrant workers are more diligent, easier to satisfy, but for the new generation, these children thirst for "quick success ". [6] Twenty years ago, Li 
Jinming managed the first generation of migrant workers in China, the only purpose for them to choose to work is to make more money to settle down in their hometown. Their level of demand is low, just to meet the basic physiological needs. However,for the new generation of migrant workers borned in 1980s and 1990s, they pursue higher income to support their city dream. Maslow's theory of demand hierarchy holds that human needs are broadly divided into five categories: physical, security, social, respect and self-realization. Five kinds of needs are ascending in order. [7] For the new generation of migrant workers, in addition to the basic physiological needs, they also have housing, medical and other security needs, as well as the need of realization of self-worth. Therefore, the "Taylor system" is no longer applicable to the new generation of migrant workers. Professor Panyi, who studies Chinese working groups for many years, believes that the new generation of migrant workers in terms of predecessors, to bear more anxiety and practical pressure, psychological activity is fierce. Thus, the analysis of the personal factors of Foxconn employees jumping suicide incident can focus on their psychological factors and realistic contrast. Mainly in the following three aspects:

\section{Conflicts between low - income and urban dreams}

For the new generation of migrant workers,the greatest dream is to settle down in the city through hard working,. However, according to the 2008 statistics showing that China's "Pearl River Delta" and "Yangtze River Delta" export factory workers's average annual income is only the two regions of the urban workers's average annual wage of 37.82\%. Thus, China's new generation of migrant workers's income and the current city comsum level, high housing prices are in sharp contrast, when the city dream broken, young people will lose the strong support to continue to struggle, accidental personal factors are more likely to be the fuse to trigger them to desperate.

\section{The Conflict of Psychological Needs and the "Tiger \& Fox" Management Mode}

"Social people" theory assume that the material benefits people get in the work, to mobilize people's work enthusiasm only secondary significance, people pay more attention to get along well with the people to work with around friendly. [8] The level of production efficiency is not determined by the working methods and working conditions, but depends on the staff "morale", and the morale depends on whether the interpersonal relationship is consistent between the family, business and social life .[9] Thus, modern management should pay attention to the sense of belonging and overall sense of the staff. However, in the "economic man" hypothesis based on the "tiger fox" management model, Foxconn ignored the the function of the emotion as a link of informal groups in the enterprise coordination and communication, so that employees cannot find emotional support and lack of psychological comfort.

\section{The conflict between the desire for collective ownership and the fragmented interpersonal state}

Famous sociologist Durkheim consider that the more isolated and alienated the individual's social relationships are ,the more likely they commit suicide. And the "collective power, is one of the most effective way to curb suicide." This shows the importance of collective power on individual behavior. Foxconn's original management model doesn's pay enough attention to the use of this power. Employee relations are in a fragmented state, and lack of communication and decompression channels, when encountering some setbacks it is easy to amplify unlimitedly negative emotions and eventually lead to tragedy.

In short, Foxconn's new generation of migrant workers personal psychological conflict grow expansion under the influence of a variety of negative social environment. According to the formula of $\mathrm{B}=\mathrm{f}(\mathrm{P} * \mathrm{E}), \mathrm{P}$ (individual) under the influence of negative $\mathrm{E}$ (environment), personal attitudes (internal force field tension) continue to tend to negative aspects, and ultimately lead to tragedy. 


\section{References}

[1] Lu Shengzhong. Management Psychology. Zhejiang: Zhejiang Education Press, 2011: 11.

[2] Liu Zhiyi, Yang Jibin. Foxconn Suicide Mystery for"Eight Jump". Southern Weekend, 2010-05-17.

[3] Chen Renfang. Foxconn "Tiger Fox" management culture model and employees "twelve jump" deep psychological conflict. Journal of Changjiang Normal University, 2011 (2).

[4] Lu Shengzhong. Management Psychology. Zhejiang: Zhejiang Education Press, 2011: 61.

[5] Fan Yijin. The Crowd Empower the Media - the top ten media events in 2010. Southern Weekend, 2010-12-17 http://www.infzm.com/content/53568.

[6] Chen Renfang. Foxconn "Tiger Fox" cultural management model and staff "twelve jump" deep psychological conflict. Journal of Changjiang Normal University, 2011 (2).

[7] Lu Shengzhong. Management Psychology. Zhejiang: Zhejiang Education Press, 2011: 141-144.

[8] Lu Shengzhong. Management Psychology. Zhejiang: Zhejiang Education Press, 2011: 63.

[9] Liang Baoyong. Management Psychology. Nankai University Press, 2006: 38. 